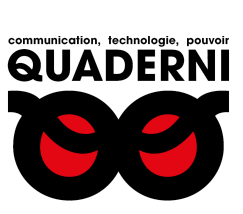

\title{
Quaderni
}

Communication, technologies, pouvoir

\section{Étudier les théories du complot en sciences sociales : enjeux et usages}

Julien Giry

\section{(2) OpenEdition}

Édition électronique

URL : https://journals.openedition.org/quaderni/1101

DOI : 10.4000/quaderni.1101

ISSN : 2105-2956

Éditeur

Les éditions de la Maison des sciences de l'Homme

Édition imprimée

Date de publication : 5 octobre 2017

Pagination : 5-11

\section{Référence électronique}

Julien Giry, «Étudier les théories du complot en sciences sociales : enjeux et usages », Quaderni [En ligne], 94 | Automne 2017, mis en ligne le 05 octobre 2018, consulté le 22 juillet 2021. URL : http:// journals.openedition.org/quaderni/1101; DOI : https://doi.org/10.4000/quaderni.1101 


\section{$D$ ossier}

\section{Étudier les théories}

du complot en

\section{sciences sociales :} enjeux et usages

\section{Julien Giry}

Docteur en science politique CNRS IREMAM Chercheur associé à IDPSP Université de Rennes 1
Ces dernières années ont vu de nombreuses théories du complot émerger de manière plus ou moins spontanée dans l'espace public à la suite des attentats de Paris ou de Bruxelles tout autant qu'au travers de discours politiquement déterminés de leaders complotistes tels que Thierry Meyssan, Alain Soral et Dieudonné M'Bala M'Bala. Aussi, les théories du complot, bien qu'anciennes, semblent concomitamment faire l'objet d'une attention, et d'une compétition analytique parfois, tout à fait inédite en France : celle des journalistes, celle des chercheurs et des scientifiques et, enfin, celle des pouvoirs publics et des professionnels de la politique.

Ainsi, la grande presse, les magazines hebdomadaires ou mensuels (L'Express, Le Point, Diplomatie, Le Monde 2 ou encore Historia) ou les télévisions (France 2 ou Canal plus) ${ }^{1}$ multiplient les sujets, les articles et les dossiers thématiques traitant de la thématique conspirationniste, notamment de l'engouement des adolescents. Les pouvoirs publics, comme en témoignent la mise en place en février $2016 \mathrm{du}$ site gouvernemental « On te manipule $»^{2}$ et la tenue du colloque Réagir face aux théories $d u$ complot $^{3}$ au muséum d'histoire naturelle à Paris, cherchent par tous les moyens à lutter contre le développement du conspirationnisme. Quant aux champs académiques français et européens, audelà de leurs pionniers, ils sont traversés par une vitalité interdisciplinaire inédite qui les rapprochent du monde anglo-saxon où les « conspiracy theories » sont un objet d'étude depuis les années 1960 au moins. Ceci se traduit par exemple par la mise en place de l'action européenne COST Comparative Analysis of Conspiracy Theories en 2016 ou la tenue d'un atelier thématique lors 
du congrès annuel de l'association française de science politique en $2017^{4}$. En outre, depuis 2010, des revues aussi hétérogènes que Science et Pseudo-science, Agone, Raison Publique, Esprit, Diogène et bientôt Émulations ont dédié un numéro au complotisme. Malgré leurs différences, ces opus se concentrent essentiellement autour de quatre thématiques classiques. $1^{\circ}$ ) La présentation des différentes théories du complot, leurs caractéristiques, leurs fonctions et les modes d'adhésion. $2^{\circ}$ ) Les aspects relatifs à la rhétorique conspirationniste. $3^{\circ}$ ) Les liens supposés ou réels entre approche critique et théories du complot ainsi que l'utilisation de ces dernières comme un anathème facile et disqualifiant. $4^{\circ}$ ) La réfutation des théories du complot.

Dès lors, dans son dossier thématique, Quaderni entend s'adresser principalement aux chercheurs en sciences humaines et sociales afin d'adopter une approche renouvelée et un angle radicalement différent de ceux habituellement mobilisés pour étudier le conspirationnisme : privilégier les liens entre complotisme, nouvelles technologies et communication. Toutefois, avant d'en venir à la question des apports de ce dossier, il convient de revenir quelque peu sur les enjeux de définition, et les usages qu'ils produisent, des notions de théories du complot ou de conspirationnisme, complotisme.

\section{« Théories du complot » et « complotisme/ conspirationnisme » : enjeux de définition, d'usages et de labellisation}

Les mots ou expressions « complotisme/iste », « conspirationnisme/iste » ou encore « théories du complot » se sont progressivement imposés dans le vocabulaire et le débat public où ils sont communément connotés de manière négative et péjorative. Ils suscitent une sorte de crainte ou de rejet instinctif envers celles ou ceux, acteurs individuels ou collectifs, qui y souscrivent ou qui sont désignés comme tels. Dès lors, ces acteurs se voient affublés d'une « labellisation infamante ${ }^{5}$ » qui, comme une étiquette, produit des effets hétéronomiques de disqualification dans divers champs sociaux parmi lesquels les champs politique, social, médiatique ou bien économique. En effet, le phénomène complotiste ne se reconnaît pas comme tel, il s'agit d'une catégorie hétérodéterminée ou d'une labellisation exogène. Dit autrement, hormis dans des dynamiques de retournement du stigmate, personne n'adhère à, ou ne défend, des «théories du complot»: certains cherchent à comprendre la « vérité vraie » derrière les faux-semblants quand d'autres tentent de découvrir les « vrais » enjeux du pouvoir par-delà les rideaux de fumée. Dans tous les cas, tous sont persuadés d'être dans une démarche individuelle salutaire, positive et bénéfique pour l'individu et la société dans son ensemble.

Dès lors, une double difficulté se pose à la fois pour les chercheurs, les journalistes et les pouvoirs publics autour des enjeux de définition, des effets qu'ils produisent et des usages qu'ils entraînent. Il s'agit d'abord de trouver une sorte de bonne mesure qui consiste à faire un bon usage, c'est-à-dire à la fois prudent et parcimonieux, des termes énoncés plus haut afin d'éviter un premier écueil qui consisterait à voir immédiatement dans toutes critiques radicales du modèle néo-libéral une forme plus ou moins explicite de complotiste $^{6}$. En d'autres termes, il ne faut pas voir des théories du complot partout, il ne faut 
pas a priori calquer cette étiquette sur tel ou tel mouvement ou individu et il est donc impératif de n'adopter ni un acception ni un usage trop extensifs de ces qualificatifs ou labels qui nous mèneraient, en particulier dans un régime dit de « démocratie-libérale », à un second écueil : l'érection de soi-même, chercheurs, journalistes ou pouvoirs publics, en police ou censeur de la pensée où « aucune pensée politique préconisant un autre mode de production, c'est-à-dire qui ne relèverait pas de la pensée dominante, $n$ 'est plus possible $e^{7}$ » qui in fine s'avérerait une démarche à la fois vaine et contre-productive. En effet, une telle démarche ne pourrait alors que renforcer, nous l'avons observé dans le cadre de «l'affaire Dieudonné $e^{8}$ » de 2014 ou de la «chasse aux sorcières » engagée contre ceux qui «n'étaient pas Charlie $e^{9}$ » début 2015, les acteurs stigmatisés dans leurs positions victimaires et leurs convictions qu'un pouvoir liberticide se met en place cherchant à faire taire les individus, à étouffer toutes critiques au détriment de la liberté d'expression et à bannir toutes formes de pratiques sociales marginales ou alternatives au profit de « bons comportements » sociaux ou politiques. À l'opposé, seul un examen ad hoc et approfondi de chacune des situations rencontrées peut permettre de déterminer si, oui ou non, nous sommes en présence de théories du complot; le qualificatif ne doit en aucun cas être déterminé ex ante en fonction de nos préférences, préjugés, clichés ou toutes autres formes de prénotions.

Dans la même veine, toute approche sérieuse du conspirationnisme doit s'affranchir des prénotions selon lesquelles le « profil-type », bien qu'il n'en existe pas ${ }^{10}$, des « adeptes du soupçon » serait un « jeune », peu doté en capital culturel (niveau de diplôme), de confession musulmane et/ou issu de l'immigration, crédule vis-à-vis de « informations » disponibles sur le net et résidant dans un quartier populaire. La quintessence de ces stéréotypes symboliques et de préjugés collectifs fut atteinte dans le reportage, Ces jeunes qui croient aux théories du complot, diffusé dans Envoyé Spécial le 29 janvier 2015, soit quelques jours après les attentats de Charlie Hebdo. En outre, il faut également se départir des approches « paranoïdes » ou « psychologisantes » qui tendent à rejeter, sans autre forme de procès, l'étude des théories du complot dans le champ des déviances psychopathologiques ou des sciences cognitives et qui interdisent de les envisager comme un fait social et politique en-soi.

En résumé, une approche authentiquement scientifique des théories du complot doit, d'une part, éviter de développer une conception trop extensive, systématique et pathologique du complotisme et, d'autre part, se garder de se poser, en fonction de préjugés ou stéréotypes symboliques, a priori et une fois pour toutes en discriminateur absolu de l'acceptable et de l'inacceptable politique ou social.

Dès lors, une fois ces précisions et/ou précautions actées, quelques éléments de définition peuvent être envisagés. Ainsi, les théories du complot traduisent d'abord la conviction profonde, à la fois déterministe et essentialiste, qu'un « groupe ou un individu omnipotent, navigant aux marges de la société, contrôle secrètement, en totalité ou en partie, l'ordre politique et social ${ }^{11} \gg$. En tant que représentation politique historicisée à la fois globale et hégémonique de la marche du monde, la pensée conspirationniste prétend qu'il existe un 
grand complot. Tous les événements extraordinaires (catastrophes naturelles, attentats ou assassinats, prouesses scientifiques ou technologiques, etc.), tous les phénomènes ayant des conséquences sociales négatives (chômage, épidémies, alcoolisme, consommation de drogues, etc.), au même titre que certains faits, situations ou circonstances en apparence insignifiants (attribution des Oscars, résultat sportif, conditionnement des denrées alimentaires, etc.), se voient présentés comme le produit de la volonté secrète, exclusive, omnipotente et omnisciente d'une minorité organisée. Le conspirationnisme produit alors des explications manichéennes ${ }^{12}$ en termes de raisons cachées, de « causalité diabolique », de liens souterrains, de forces occultes, de pactes et d'agendas secrets affirmant lever le voile sur une réalité prétendument dissimulée aux yeux de tous. Concrètement, la démarche conspirationniste s'attache à recueillir et à ordonner, à l'intérieur d'une trame narrative unique et cohérente, des faits et des événements épars qui a priori ne font pas sens entre eux. Pardelà leur signification apparente, l'intention est alors d'apporter la preuve que les faits et les événements en question sont forcément liés entre eux, car ils résultent d'une cause unique, c'est-à-dire d'un complot dont ils témoignent. En d'autres termes, un conspirationniste ne se contente pas de dénoncer tel ou tel complot, réel ou fantasmé. Bien au contraire, le complot devient la grille systématique et systémique à travers laquelle l'ensemble de l'histoire humaine est lue et interprétée ${ }^{13}$. Aussi, si on le veut résumer par une expression paradoxale, il convient d'affirmer haut et fort que, si «les complots existent; le complot n'existe pas ${ }^{14} »$. En effet, nul ne saurait ignorer que des complots authentiques existent bel et bien, mais nul ne doit non plus affirmer que parce que ou tel complot est historiquement avéré, il doit nécessairement et/ ou immédiatement servir de précédant légitimelégitimant à/pour telle ou telle théorie du complot. Dit concrètement, ce n'est pas parce qu'il y a eu le scandale du Watergate que le 11 septembre est de facto un « inside job » ou le fruit d'un complot de « l'État profond» américain. En conséquence, ce qui emporte la labellisation complotiste, c'est le systématisme ou le systémisme qui veut que le complot devienne le moteur unique de l'histoire et la seule grille de lecture qui vaille des phénomènes sociaux : «Tout est lié. Rien n'arrive par hasard. Les choses ne sont pas ce qu'elles semblent être ${ }^{15} »$. On se retrouve alors face à un paradoxe inextricable au sein duquel rien ne peut être tenu pour acquis (hyper-critique), tout n'est que faux-semblants, rideaux de fumée et théâtre d'ombres, si ce n'est l'hypothèse irréfragable du complot (infra-critique) qui débouche irrémédiablement sur la formulation d'une pseudo-critique accusatoire.

\section{Une approche renouvelée de l'étude des théories du complot en sciences sociales}

Compte tenu de l'ensemble des éléments avancés jusqu'ici, ce dossier thématique s'assigne a minima un double objectif. D'une part, il envisage les théories du complot comprises comme un fait social et politique en-soi, et d'autre part, il porte une attention toute particulière à son déploiement sur et au travers des moyens et réseaux de communication modernes. Ceci implique d'abord une approche objectivée ou réifiée des théories du complot qui rejette à la fois la vision selon laquelle elles constitueraient une sorte de pathologie collective ou de " paranoïa » du corps social et relèveraient, en conséquence, d'un exceptionnalisme méthodologique. Tout à l'inverse, comme le 
montrent P. France et A. Motta dans leur étude du militantisme au sein de l'association ReOpen 911, il est nécessaire et indispensable d'appliquer à l'étude du phénomène complotiste les outils classiques des sciences sociales et de partir de données empiriques. De même, c'est dans une démarche processuelle ou interactionniste que S. Alava, H. Hussein et $\mathrm{N}$. Nojjar envisagent comment les théories du complot jouent un rôle essentiel et décisif dans les trajectoires ou les carrières de radicalisation islamistes et djihadistes sur l'internet. De l'internet, il en sera alors question dans les contributions de J. Giry et I. Yablokov. La première envisage une étude de cas d'une vidéo recombinante (found footage), " Alain Soral sauve Glenn et Tara dans The Walking Dead», qui illustre la manière dont le tournant vidéo du médiactivisme a pu être investi afin de produire à peu de frais des discours et des contenus conspirationnistes esthétisés en phase avec les dispositifs sociotechniques qui les hébergent et la « culture jeune ». De « culture jeune » et des craintes qu'elle suscite, il en sera enfin question dans l'article de I. Yablokov qui éclaire la construction publique et la dynamique d'une « panique conspirationniste » dans la Russie contemporaine autour de rumeurs de suicides d'adolescent(e)s.

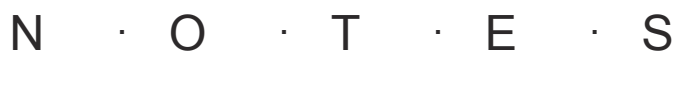

1. Par exemple : «Spécial investigation - 11 septembre : enquête sur la théorie du complot ", Canal plus, 11 septembre 2009. « Spécial investigation - Enquête sur le réseau Dieudonné », Canal plus, 5 juillet 2014. «Envoyé spécial - Charlie Hebdo : ces jeunes qui soutiennent la théorie du complot », France 2, 29 janvier 2015. « Envoyé spécial - Les adeptes du soupçon », France 2, 7 janvier 2016.

2. En ligne : http://www.gouvernement.fr/on-temanipule Accédé : 16 juin 2017. Du reste, dès le lendemain, un site miroir à l'iconographie similaire, on-te-manipule.com, a été mis en place afin de dénoncer les authentiques manipulations étatiques, les « coups fourrés » des pouvoirs publics et leur volonté de s'ériger en police politique de la pensée unique. En ligne : http://on-te-manipule.com/ Accédé : 05 août 2017.

3. En ligne : http://cache.media.education.gouv.fr/ file/02 - fevrier/50/6/Programme de la journee d etude_Reagir_face_au_theories_du_complot_535506. pdf Accédé : 18 juillet 2017

4. En ligne : http://www.afsp.info/congres/ congres-2017/sessions/sections-thematiques/st52/ Accédé : 18 juillet 2017

5. Emmanuel Taïeb, «Logiques politiques du conspirationnisme », Sociologie et sociétés, vol.42, $\mathrm{n}^{\circ} 2,2010$, p. 268.

6. Ces travers, nous les retrouvons notamment dans les travaux de P.-A. Taguieff qui s'inscrivent dans la grille hofstadterienne selon laquelle : critique sociale radicale - des dominants $-\mathrm{du}$ système néolibéral $=$ populisme $=$ conspirationnisme $=$ antisémitisme . Richard Hofstadter, The Age of Reform, New York, Vintage Books, 1955. Pierre-André Taguieff, Court traité de complotologie, Paris, Mille et une nuits, 2013. 
7. Maurice Duval, « Des peurs collectives : le discours anti-secte comme support de l'idéologie néo-libérale », L'Homme et la société, vol.1, n¹55, p. 67.

8. Sur la notion d' " affaire », voir : Nicolas Offenstadt et Stéphane Van Damme (dir.), Affaires, scandales et grandes causes, Paris, Stock, 2007.

9. On se souviendra ainsi des mots sans ambiguïté prononcés par l'éditocrate de France 2 Nathalie Saint-Cricq lors du journal télévisé du 12 janvier 2015 : «C'est ceux qui ne sont pas Charlie qu'il faut repérer, ceux qui dans certains établissements scolaires ont refusé la minute de silence, ceux qui balancent sur les réseaux sociaux et ceux qui ne voient pas en quoi ce combat est le leur. Et bien, ce sont eux que nous devons repérer, traiter, intégrer ou réintégrer dans la communauté nationale. Et là, l'École et les politiques ont une lourde responsabilité ». En ligne : https://www.youtube.com/watch?v=wW3vil_cJ7I Accédé : 10 août 2017. Dans la même veine, un petit garçon de 8 ans en classe de CE2 a été placé sous régime d' " audition libre » au commissariat de Nice pour « apologie de terrorisme ». L'enfant aurait ainsi dit à son instituteur ne pas être Charlie à cause des caricatures du prophète. Sylvain Mouillard, « un enfant de 8 ans au commissariat pour "apologie du terrorisme" », Libération, 28 janvier 2015.

10. Par exemple : Anita M. Waters, « Conspiracy Theories as Ethnosociologies. Explanation and Intention in African Americain Political culture », Journal of Black Studies, vol.28, n 1, 1997, pp. 112125. Veena Das, « Specificities: Official Narratives, Rumor, and the Social Production of Hate. Social Identities », Journal for the Study of Race, Nation and Culture, vol.4, $\mathrm{n}^{\circ} 1$, 1998, pp. 109-130. William Simmons, Sharon Parsons, « Beliefs in Conspiracy Among African Americans : A Comparaison of Elites and Masses », Social Science Quarterly, vol.86, n³, 2005, pp. 582-598. Julien Giry, Le conspirationnisme dans la culture politique et populaire aux États-Unis. Une approche sociopolitique des théories du complot, Thèse de doctorat en science politique, Université de Rennes 1, 2014.

11. Mark Fenster, Conspiracy theories : Secrecy and Power in American Culture [1999], Minneapolis, University of Minneapolis, 2008, p. 1. Pour Taïeb, « on peut identifier la posture conspirationniste, quand son discours postule que le cours de l'Histoire et les événements marquants qui la jalonnent sont provoqués uniformément par l'action secrète d'un petit groupe d'hommes désireux de voir la réalisation d'un projet de contrôle et de domination des populations ». Emmanuel Taïeb, art cit., p. 267.

12. Pour Hall, et nous partageons ce point de vue, ce qui caractérise le conspirationnisme, c'est précisément cette tendance des acteurs à interpréter systématiquement les faits sociaux au travers d'une opposition « nous »/ « eux » dont le premier terme est toujours la victime innocente et passive tandis que, invariablement, le second est le responsable et l'organisateur exclusif de tous les maux. J. A. Hall, «Aligning Darkness with Conspiracy Theory : The Discursive Effect of African American Interest in Gary Webb's "Dark Alliance" ", The Howard Journal of Communication, vol.17, n³, 2006, pp. 205-222.

13. «Un adepte des théories de la conspiration n'est pas seulement quelqu'un qui admet l'existence de certaines conspirations particulières. Il est plutôt quelqu'un qui opère à partir d'une approche méthodologique générale et d'un ensemble de priorités. Quand ils $s$ 'efforcent de comprendre les événements, les adeptes des théories de la conspiration vont à la recherche de groupes agissant en secret, en dehors des règles institutionnelles établies, de façon dévoyée, ou en vue de manipuler les sentiments du public afin de faire porter le chapeau à d'autres parties. Les adeptes des théories de la conspiration mettent l'accent sur 
les méthodes des conspirateurs, leurs motifs et leurs effets. Les personnalités, les horaires personnels, les rencontres secrètes et les actions conjointes des conspirateurs occupent le centre de leur attention". Michael Albert, Stephen Shalom, «Conspirations ou institutions ? Le 11 Septembre et au-delà », Agone, $\mathrm{N}^{\circ} 47,2002$, p. 31.

14. Alain Goldschläger, Jacques-Charles Lemaire, Le complot judéo-maçonnique, Bruxelles, Éditions Labor, 2005, p. 7.

15. Véronique Campion-Vincent, La société parano. Théories du complot, menaces et incertitudes [2005], Paris, Petite Bibliothèque Payot, 2007, p. 11. 
